\title{
The Correlation between Uric Acid Levels and Amyotrophic Lateral Sclerosis
}

\author{
Yu-Wei Lin ${ }^{1, *}$, Thy-Sheng Lin ${ }^{2}$, Ming-Liang Lai ${ }^{2}$ \\ ${ }^{1}$ Department of Neurology, Mackay Memorial Hospital, Taiwan \\ ${ }^{2}$ Department of Neurology, National Cheng Kung University Hospital, Taiwan \\ *Corresponding author: linyw @ kscg.url.com.tw
}

Received April 30, 2013; Revised May 15, 2013; Accepted May 16, 2013

\begin{abstract}
Introduction Uric acid (UA) may act as an antioxidant in ALS pathophysiology. This study examined whether serum UA concentration correlates to clinical course in the ALS patients. Methods We measured serum UA levels in 51 sporadic ALS patients, and compared them to 51 age-and-gender-matched healthy subjects. We analyzed the correlation between serum UA levels, illness duration, and dis ease "course factors" (defined as "disease duration" over "terminal time") in the ALS patients. Results The mean serum UA levels in the ALS groups were higher than the control groups in both genders. Serum UA levels were inversely correlated with the disease "course factors" in the ALS male patients. Discussion The elevation of serum UA concentration in ALS patients may be related to oxidative stress. The reasons that serum UA levels inversely correlated with ALS "course factors" include: muscle wasting, poor nutrition, reduced antioxidant reserve, or increased UA consumption in end-stage patients.
\end{abstract}

Keywords: amyotrophic lateral sclerosis, uric acid, oxidative stress, SOD1, TDP-43

\section{Introduction}

The pathophysiology of amyotrophic lateral sclerosis (ALS) has been postulated to be related to oxidative stress from glutamate-mediated excitotoxicity, especially since the discovery of $\mathrm{Cu} / \mathrm{Zn}$ superoxide dis mutase (SOD) gene mutations in familial ALS and TDP-43 aggregation in sporadic ALS patients [1]. Recent findings suggest that glutamate-mediated NO-overproduction and the subsequent generation of peroxynitrite play an important role in the pathogenesis of ALS [1]. Glutamate-mediated excitotoxicity is associated with adenosine triphosphate degradation, which is reflected by increased levels of extracellular hypoxanthine and xanthine and the generation of oxygen radicals. As xanthine is degraded to uric acid, it has been shown that elevated levels of both xanthine and uric acid in the cerebrospinal fluid (CSF) are related to glutamate-mediated excitotoxicity in some neurological disorders [1].

Uric acid (UA) has the ability to react with peroxynitrite and stimulate the expression of extracellular SOD which may confer additional antio xidant protection, and has therapeutic action in mouse model of experimental autoimmune encephalomyelitis (EAE) by inactivating $\mathrm{ONOO}(-)$, preventing blood-brain barrier breakdown, and reducing neuroimmune responses [1]. It also stabilizes ascorbate, possibly by forming complexes with iron ions, and scavenges nitrogen radicals [2].

There is a correlation between serum urate and CSF urate in humans with an intact blood-brain barrier, and the CSF urate concentration is approximately 7 10\% that of plasma $[3,4]$. Considering an intact blood-brain barrier in
ALS patients and healthy persons, a comparis on of serum UA levels may be able to reflect the CSF UA levels in these two groups.

We hypothesized that the concentration of uric acid would be elevated in ALS patients, and may have a correlation to the disease course or severity. Therefore, we aimed to determine whether the concentration of serum UA level correlated to the clinical parameters and disease course in ALS patients.

\section{Methods}

\subsection{Study Population}

We enrolled patients diagnosed with ALS at National Cheng Kung University Hospital and Mackay Memorial Hospital, from 1996/9 to 2006/9. All subjects met the EI Escorial World Federation of Neurology criteria for the diagnosis of ALS. We excluded the patients with chronic kidney disease, diabetes mellitus, gout, and those who were receiving any medications that would affect serum UA levels.

\subsection{Data Collection}

We retrospectively reviewed chart records for serum UA data measured at the time of diagnosis, during hospitalization, and outpatient follow-up. The onset time of ALS, the illness duration at the time of blood sampling, and illness terminal time (meaning the time of death from the disease) were recorded. We also collected serum UA data of 51 age- and gender-matched healthy subjects (the control group). 


\subsection{Data Analysis}

We use the matched-pairs t-test to compare the serum UA data of the ALS patients at the time they were diagnosed to the serum UA data of the control group. Using serum UA levels from the ALS patients at different durations of illness, we performed regression analysis to correlate serum UA levels with illness duration, disease stage, and progression rate. We definite "illness duration/terminal time" as "course factors", and also correlated serum UA levels with the course factors.

\section{Results}

Fifty-one ALS patients (38 male patients, 13 female patients) with serum UA data at the time of diagnos is from two medical centers were included in our analysis. All of these patients were in the early stage of ALS (according to the definition by the Augmentative and Alternative Communication Institute, AAC) at the time they were diagnosed. We divide the ALS patients and control group into male and female groups, and compared the serum UA levels separately. The average serum UA level in the male ALS patients was $6.489 \mathrm{mg} / \mathrm{dL}$ compared to $5.961 \mathrm{mg} / \mathrm{dL}$ in the male controls (Table 1), and $5.508 \mathrm{mg} / \mathrm{dL}$ in the female ALS patients compared to $5.054 \mathrm{mg} / \mathrm{dL}$ in the female controls (Table 2). There were significantly higher mean serum UA levels in the ALS patients compared to the control group, both in males and females.
Table 1. Comparing a verage UA le vel of ALS male patien ts to health male group

\begin{tabular}{|c|c|c|c|c|}
\hline \multicolumn{5}{|c|}{ One Sample t-test } \\
\hline & Mean & DF & t-Value & P-Value \\
\hline $\begin{array}{c}\text { health } \\
\text { male }\end{array}$ & 5.961 & 37 & 39.429 & $<.0001$ \\
\hline ALS male & 6.489 & 37 & 26.535 & $<.0001$ \\
\hline
\end{tabular}

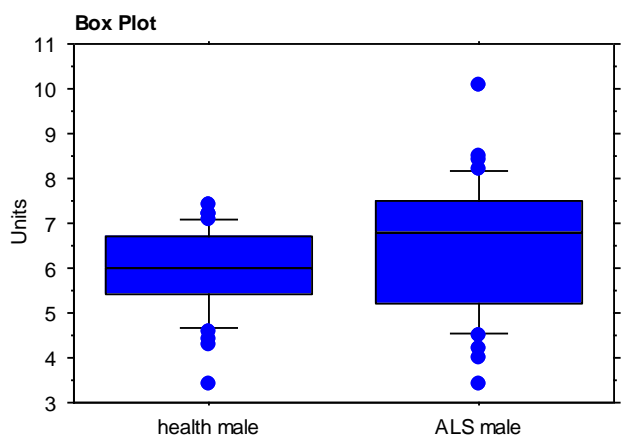

Figure 1. The graph shows higher average UA level of ALS male patients comparing to health male group. Y-axis represents UA level (unit: $\mathrm{mg} / \mathrm{dL}$ )

Table 2. Comparing average UA le vel of ALS female patients to heal th female group

\begin{tabular}{|c|c|c|c|c|}
\hline \multicolumn{5}{|c|}{ One Sample t-test } \\
\hline & Mean & DF & t-Value & P-Value \\
\hline $\begin{array}{c}\text { health } \\
\text { female }\end{array}$ & 5.054 & 12 & 20.296 & $<.0001$ \\
\hline $\begin{array}{c}\text { ALS } \\
\text { female }\end{array}$ & 5.508 & 12 & 13.745 & $<.0001$ \\
\hline
\end{tabular}

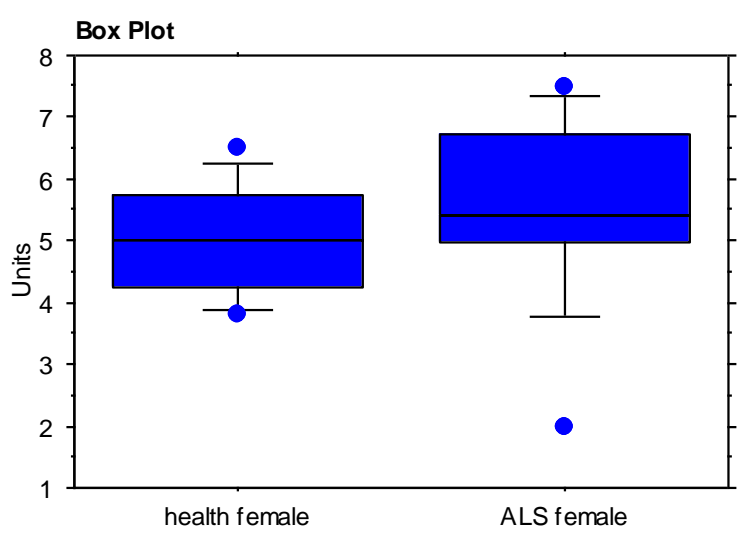

Figure 2. The graph shows higher average UA level of ALS female patients comparing to health female group. Y-axis represents UA level (unit: $\mathrm{mg} / \mathrm{dL}$ )

Using serum UA data from the ALS patients at different durations of illness, we correlated the serum UA data to illness duration and "course factors" (i.e. illness duration/terminal time). As the disease progressed, serum UA levels gradually declined, eventually reaching below the normal range of serum UA levels in the controls when the patients progressed to the terminal stage. The average serum UA level in late stage male ALS patents was about $3 \mathrm{mg} / \mathrm{dL}$. In the male ALS patients, the serum UA level was inversely correlated with the course factors $\left(\mathrm{R}^{2}: 0.677\right)$ (Table 3), but not with the illness duration $\left(\mathrm{R}^{2}: 0.166\right)$ (Table 4). In the female ALS patients, because the sample size was too small, the tendency of UA levels was not significantly correlated with either illness duration (Table 5) or course factors (Table 6).
Table 3. The correlation between UA levels an d "course factors" (du ration/terminal) in ALS male patients

\begin{tabular}{|c|c|c|c|c|c|}
\hline \multicolumn{6}{|c|}{$\begin{array}{c}\text { Regression Summary } \\
\text { UA male vs. M Duration/Terminal }\end{array}$} \\
\hline \multicolumn{3}{|c|}{ Count } & \multicolumn{3}{|c|}{42} \\
\hline \multicolumn{3}{|c|}{ Num Missing } & \multicolumn{3}{|c|}{19} \\
\hline \multicolumn{3}{|c|}{$\mathrm{R}$} & \multicolumn{3}{|c|}{.823} \\
\hline \multicolumn{3}{|c|}{ R Squared } & \multicolumn{3}{|c|}{.677} \\
\hline \multicolumn{3}{|c|}{ Adjusted R Squared } & \multicolumn{3}{|c|}{.669} \\
\hline \multicolumn{3}{|c|}{ RMS Residual } & \multicolumn{3}{|c|}{1.105} \\
\hline \multicolumn{6}{|c|}{$\begin{array}{c}\text { ANONATable } \\
\text { UA male vs. M Duration/T erminal } \\
\end{array}$} \\
\hline & DF & $\begin{array}{l}\text { Sum of } \\
\text { Squares }\end{array}$ & $\begin{array}{l}\text { Mean } \\
\text { Square }\end{array}$ & $\begin{array}{c}\text { F- } \\
\text { Value }\end{array}$ & P-Value \\
\hline Regression & 1 & 102.339 & 102.339 & 83.803 & $<.0001$ \\
\hline Residual & 40 & 48.847 & 1.221 & & \\
\hline Total & 41 & 151.186 & & & \\
\hline
\end{tabular}




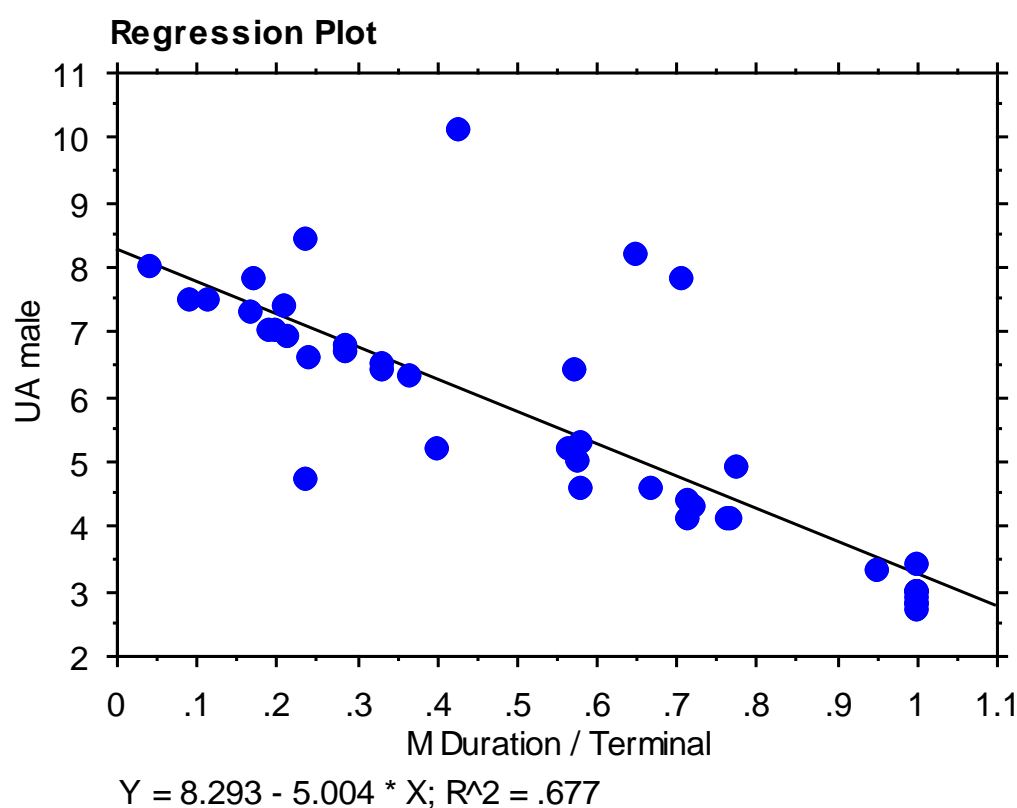

Figure 3. The graph indicates the UA levels decline with "course factors" (duration/terminal) in ALS male patients $\left(\mathrm{r}^{2}=0.677\right)$. X-axis represents the "course fact ors" (duration/terminal) while UA levels were measured. Y-axis represents UA levels (unit: mg/dL)

Table 4. The correlation between UA le vels andillness du ration in ALS male patients

\begin{tabular}{|c|c|}
\hline \multicolumn{2}{|c|}{$\begin{array}{c}\text { Regression Summary } \\
\text { UA male vs. M Duration }\end{array}$} \\
\hline Count & 61 \\
\hline Num Missing & 0 \\
\hline R & .408 \\
\hline R Squared & .166 \\
\hline Adjusted R Squared & .152 \\
\hline RMS Residual & 1.661 \\
\hline
\end{tabular}

\begin{tabular}{|c|c|c|c|c|c|}
\hline \multicolumn{7}{|c|}{ ANONATable } \\
\hline & DF & $\begin{array}{c}\text { Sum of } \\
\text { Squares }\end{array}$ & $\begin{array}{c}\text { Mean } \\
\text { Square }\end{array}$ & F-Value & $\begin{array}{c}\text { P- } \\
\text { Value }\end{array}$ \\
\hline Regression & 1 & 32.491 & 32.491 & 11.772 & .0011 \\
\hline Residual & 59 & 162.840 & 2.760 & & \\
\hline Total & 60 & 195.331 & & & \\
\hline
\end{tabular}

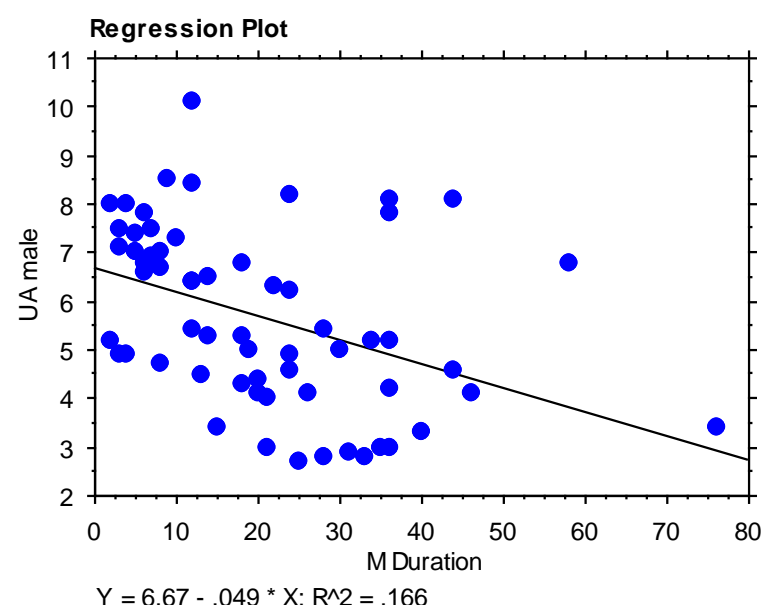

Figure 4. The graph shows no significant correlation between UA levels and illness duration in ALS male patients. $\mathrm{X}$-axis represents the durations while UA levels were measured. Y-axis represents UA levels (unit: $\mathrm{mg} / \mathrm{dL}$ )
Table 5. The correlation between UA le vels andillness duration in ALS female patients

\begin{tabular}{|c|c|}
\hline \multicolumn{2}{|c|}{ Regression Summary } \\
UA male vs. F Duration \\
\hline Count & 16 \\
\hline Num Missing & 0 \\
\hline R & .264 \\
\hline R Squared & .070 \\
\hline Adjusted R Squared & .004 \\
\hline RMS Residual & 1.652 \\
\hline
\end{tabular}

ANONATable

UA male vs. F Duration

\begin{tabular}{|c|c|c|c|c|c|}
\hline & DF & $\begin{array}{c}\text { Sum of } \\
\text { Squares }\end{array}$ & $\begin{array}{c}\text { Mean } \\
\text { Square }\end{array}$ & $\begin{array}{c}\text { F- } \\
\text { Value }\end{array}$ & $\begin{array}{c}\text { P- } \\
\text { Value }\end{array}$ \\
\hline Regression & 1 & 2.874 & 2.874 & 1.053 & .3222 \\
\hline Residual & 14 & 38.220 & 2.730 & & \\
\hline Total & 15 & 41.094 & & & \\
\hline
\end{tabular}

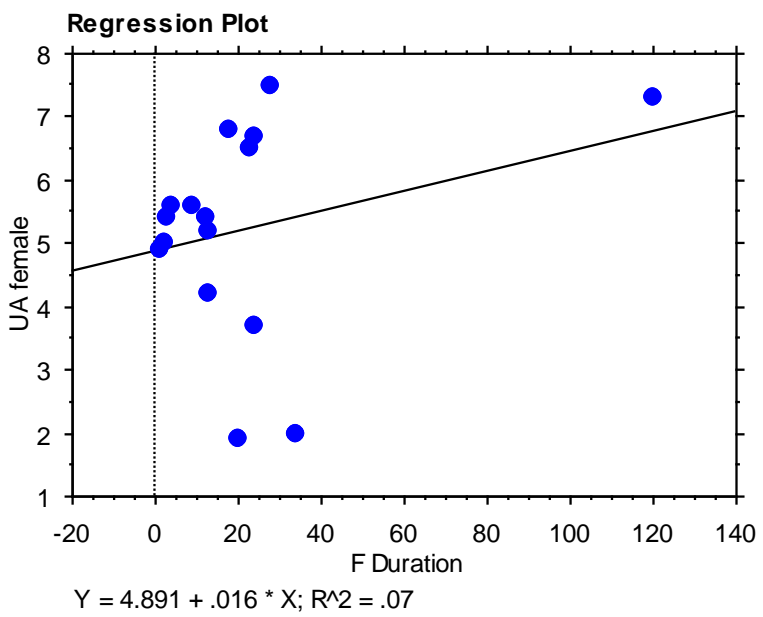

Figure 5. The graph shows no significant correlation between UA levels and illness duration in ALS female patients. $\mathrm{X}$-axis represents the durations while UA levels were measured. Y-axis represents UA levels (unit: $\mathrm{mg} / \mathrm{dL}$ ) 
Table 6. The correlation between UA le vels an d "course factor" (du ration/terminal) in ALS female patients

\begin{tabular}{|c|c|c|c|c|c|}
\hline \multicolumn{6}{|c|}{$\begin{array}{c}\text { Regression Summary } \\
\text { UA male vs. Some of F Duration/T erminal }\end{array}$} \\
\hline \multicolumn{3}{|c|}{ Count } & \multicolumn{3}{|c|}{7} \\
\hline \multicolumn{3}{|c|}{ Num Missing } & \multicolumn{3}{|c|}{9} \\
\hline \multicolumn{3}{|c|}{$\mathrm{R}$} & \multicolumn{3}{|c|}{.809} \\
\hline \multicolumn{3}{|c|}{ R Squared } & \multicolumn{3}{|c|}{.654} \\
\hline \multicolumn{3}{|c|}{ Adjusted R Squared } & \multicolumn{3}{|c|}{.585} \\
\hline \multicolumn{3}{|c|}{ RMS Residual } & \multicolumn{3}{|c|}{1.095} \\
\hline \multicolumn{6}{|c|}{$\begin{array}{c}\text { ANONATable } \\
\text { UA female vs. Some of F Duration/Terminal }\end{array}$} \\
\hline & DF & $\begin{array}{l}\text { Sum of } \\
\text { Squares }\end{array}$ & $\begin{array}{l}\text { Mean } \\
\text { Square }\end{array}$ & F-Value & $\begin{array}{c}\text { P- } \\
\text { Value }\end{array}$ \\
\hline Regression & 1 & 11.353 & 11.353 & 9.467 & .0276 \\
\hline Residual & 5 & 5.996 & 1.199 & & \\
\hline Total & 6 & 17.349 & & & \\
\hline
\end{tabular}

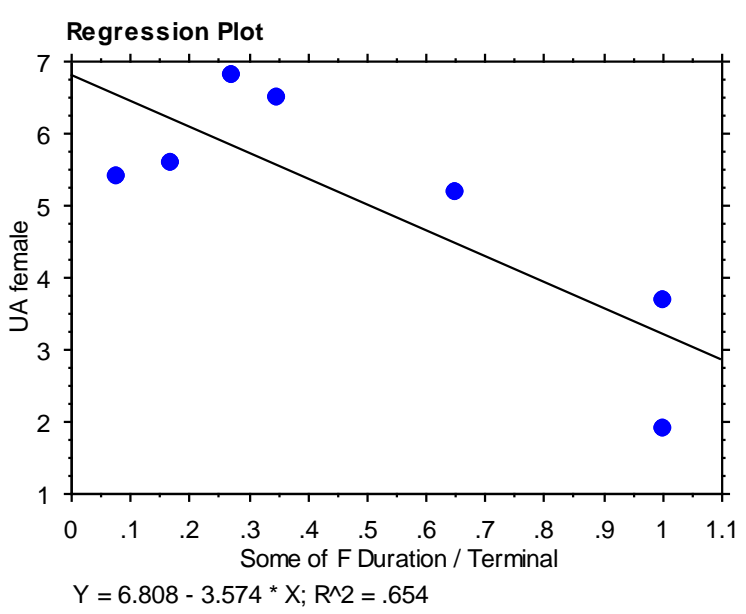

Figure 6. The graph indicates the UA levels decline with "course factor" (duration/terminal) in ALS female patients $\left(r^{2}=0.654\right) . \mathrm{X}$-axis represents the "course factor" (duration/terminal) while UA level was measured. Yaxis represents UA level (unit: $\mathrm{mg} / \mathrm{dL}$ )

\section{Discussion}

According to the AAC Institute, the symptoms of early stage ALS include limb weakness and loss of muscle mass, while ambulatory and most daily functions are preserved. In this study, we found that in patients with the early stage of ALS, serum UA levels were significantly higher compared to the controls. However, as the disease progressed, serum UA levels decreased, and in patients with late stage ALS, the serum UA levels were significantly lower than the normal range. The difference in serum UA levels between the early and late stage was $3.5 \mathrm{mg} / \mathrm{dL}$ in average.

We found that the serum UA level of male ALS patients was not correlated to the duration of disease, but was correlated with the course factors of disease. Using regression analysis, we divided the full course of male ALS patients into three intervals: 1 ) course factors (illness duration/terminal time) less than $0.25 ; 2$ ) course factors between 0.25 0.75; and 3) course factors greater than 0.75 . The average serum UA level of male ALS patients in the first interval was above $6 \mathrm{mg} / \mathrm{dL}$, and below $4 \mathrm{mg} / \mathrm{dL}$ in the last interval. At the end point of the disease course (meaning illness duration/terminal time $=1$ ), the serum UA levels of the male ALS patients were below the lower limit of normal values. Regression analysis of the female
ALS patients did not show a significant correlation between UA levels and course factors; however we found that there was a similar tendency in the UA levels of the female patients as with the male patients, according to the limited data.

In subjects without ALS, the strongest correlates of serum urate level were male sex, obesity, and arterial hypertension [2]. Use of thiazide diuretics or nonsteroidal anti-inflammatory drugs, and cigarette smoking are known to increase urate levels [2]. However, the relationship between serum urate level and ALS progression in our study was independent from these factors.

According to our results, although the average serum UA level in the early stage ALS patients was higher than in the controls, we found that the prevalence rates of gout and cardiovascular events were not elevate compared to the controls. This suggests that the elevation of serum UA may be a reactive process to the increasing oxidative stress in early stage ALS patients.

Many studies have reported a relationship between UA level and free radical/oxidant scavengers. Some studies have also demonstrated a correlation between serum UA concentration and certain disorders of the central nervous system, including multiple sclerosis (MS) and Parkinson's disease (PD), and strongly suggest that the antioxidant effect of urate is important in protecting against MS and PD $[2,5,6,7,8,9,10]$. Un like MS and PD, in which serum UA levels are low from the beginning and do not change much through the course of disease, the UA levels in ALS patients are higher in the early stage and then decrease through the course. The UA levels reach the lowest point in the terminal stage of ALS, and are far lower than in MS and PD patients.

Many studies on the pathogenes is of ALS have focused on the properties of SOD1 mutations, which contribute to about $20 \%$ of familial ALS (FALS) cases. It has been proved that mutations in the SOD1 gene cause toxic gains of function of abnormal $\mathrm{Cu} / \mathrm{Zn} \mathrm{SOD}$ enzyme, lead to increase in peroxidase activity in the FALS patients $[11,12]$. Mutant SOD1 may also accept peroxynitrites or hydrogen peroxide as a substrate, and thereby catalyze the nitration of tyrosine residues of SOD1 and hydroxyradicals [13], increasing oxidative stress in the FALS patients.

Recent studies have placed emphasis on the accumulation of abnormal TDP-43 protein in the majority of sporadic ALS cases. Chronic excitotoxicity, oxidative stress, proteas ome dysfunction and endoplasmic reticulum stress mechanistically induce mislocalization, phosphorylation and aggregation of TDP-43 [14]. Mutant TDP-43 can induce mitochondrial dysfunction, oxidative damage and nuclear accumulation of nuclear factor E2related factor 2 [15]. Studies on abnormal TDP-43expressing cells have also shown markedly increased markers of oxidative stress, apoptosis, and necros is [16].

Urate, the product of xanthine oxidation, is a good reductant and effective at preventing peroxynitrite from nitrating the tyrosine residues of proteins, thereby preventing the inactivation of cellular enzymes and modification of the cytoskeleton [17]. The ability of UA to preferentially react with peroxynitrite has been shown to aid in substantial antioxidant benefits, including the stabilization of endothelial nitric oxide synthase (eNOS) activity [6]. UA can also chelate metal ions such as iron 
and copper, converting them to poorly reactive forms unable to catalyze free-radical reactions ${ }^{18}$. In vitro and in vivo studies suggest that UA also exerts an antioxidant effect on neurons $[8,9,12,19]$.

Serum uric acid can be readily increased by diet, particularly fatty meats rich in purines, alcohol (especially beer and hard liquor) and fructose. Low-fat dairy products have the opposite effect, which may be mediated by the ability of lactose (and galactose) to activate UA transferase [6,17]. Milk has been found to lower serum UA levels by increasing UA excretion by the kidneys [17,18]. High consumption of meat, a food rich in purine, may cause hyperuricemia [17,20]. Exercise increases serum UA levels and decreases urinary excretion of UA. The inverse correlation between subsequent serum UA levels and disease stages may have been caused by muscle wasting, decreased exercise, and low meat intake of the ALS patients. Reduced serum UA levels may represent a constitutive loss of protection against nitric oxide and its oxidizing congeners, and reduced antioxidant reserve. In addition, increased UA consumption as a oxidant scavenger may also have influenced the serum UA levels as the disease progressed. Furthermore, the rate of progression to the primary clinical end point declined with increasing levels of baseline seru murate.

Overall, it seems unlikely that the inverse relationship between UA levels and ALS progression was due to confounding by known factors. We analyzed the data in a number of ways, including controlling for confounding factors, such as age, gender, kidney disease, and medications that influence UA levels.

The strength of our study is that we collected UA data at different time points in the same patients, enabling us to analyze the correlation between UA levels and disease course. This correlation has not been studied previously by any other articles in the literature. The tendency of UA levels through the disease course is a significant finding in studying the mechanism of ALS.

The limitations of this study include that we did not monitor or restrict the daily diet of our ALS patients. In addition, we did not follow up and adjust the patients' body mass index data, and the effect of riluzole treatment was not considered. To determine whether or not riluzole or other experimental medications increase serum UA levels, double-blind prospective placebo controlled studies are needed with a greater nu mber of patients.

\section{Abbreviations}

$\begin{array}{ll}\text { ALS: } & \text { amyotrophic lateral sclerosis } \\ \text { UA: } & \text { uric acid } \\ \text { SOD: } & \text { superoxide dis mutase } \\ \text { CSF: } & \text { cerebrospinal fluid } \\ \text { EAE: } & \text { experimental autoimmune encephalomyelitis } \\ \text { AAC: } & \text { the Augmentative and Alternative } \\ & \text { Communication Institute } \\ \text { MS: } & \text { multiple sclerosis } \\ \text { PD: } & \text { Parkinson's disease } \\ \text { FALS: } & \text { familial amyotrophic lateral sclerosis } \\ \text { TDP-43: } & \text { TAR DNA-binding protein-43 } \\ \text { eNOS: } & \text { endothelial nitric oxide synthase }\end{array}$

\section{References}

[1] Kokić AN, Stević Z, Stojanović S, Blagojević DP, Jones DR, Pavlović S, et al. Biotransformation of nitric oxide in the cerebrospinal fluid of amyotrophic lateral sclerosis patients. Redox Rep 2005; 10(5):265-270.

[2] Schwarzschild MA, Schwid SR, Marek K, Watts A, Lang AE, Oakes D, et al. Serum urate as a predictor of clinical and radiographic progression in Parkinson disease. Arch Neurol 2008 65(6):716-723.

[3] Láhoda F, Athen D. Typing of uric acid level in cerebrospinal fluid in neurological and psychiatric disease. Adv Exp Med Biol 1977; 76B:256-258.

[4] Niklasson F, Agren H. Brain energy metabolism and blood-brain barrier permeability in depressive patients: analyses of creatine, creatinine, urate, and albumin in CSF and blood. Biol Psychiatry 1984; 19(8):1 183-1206.

[5] Rentzos M, Nikolaou C, Anagnostouli M, Rombos A, T sakanikas $\mathrm{K}$, Economou $\mathrm{M}$, et al. Serum uric acid and multiple sclerosis. Clin Neurol Neurosurg 2006; 108(6):527-531.

[6] Sánchez-Lozada LG, Nakagawa T, Kang DH, Feig DI, Franco M Johnson RJ, et al. Hormonal and cytokine effects of uric acid. Curr Opin Nephrol Hypertens 2006; 15(1):30-33.

[7] Schlesinger I, Schlesinger N. Uric acid in Parkinson's disease. Mov Disord 2008; 23(12):1653-1657.

[8] Spitsin SV, Scott GS, Kean RB, Mikheeva T, Hooper DC Protection of myelin basic protein immunized mice from freeradical mediated inflammatory cell invasion of the central nervous system by the natural peroxynitrite scavenger uric acid. Neurosci Lett $2000 ; 292(2): 137-141$.

[9] Kean RB, Spitsin SV, Mikheeva T, Scott GS, Hooper DC. The peroxynitrite scavenger uric acid prevents inflammatory cell invasion into the central nervous system in experimental allergic encephalomyelitis through maintenance of blood-central nervous system barrier integrity. J Immunol 2000; 165(11):6511-6518.

[10] Mattle HP, Lienert C, Greeve I. Uric acid and multiple sclerosis. Ther Umsch 2004; 61(9):553-555.

[11] Spitsin SV, Scott GS, Kean RB, Mikheeva T, Hooper DC. Protection of myelin basic protein immunized mice from freeradical mediated inflammatory cell invasion of the central nervous system by the natural peroxynitrite scavenger uric acid. Neurosci Lett 2000; 292(2):137-141.

[12] Wiedau-Pazos M, Goto JJ, Rabizadeh S, Gralla EB, Roe JA, Lee MK, et al. Altered Reactivity of Superoxide Dismutase in Familial Amyotrophic Lateral Sclerosis. Science 1996; 271 :515-518.

[13] Pasinelli P, Brown RH. Molecular biology of amyotrophic lateral sclerosis: insights from genetics. Nat Rev Neurosci 2006; 7(9):710-723.

[14] Ayala V, Granado-Serrano AB, Cacabelos D, Naudí A, Ilieva EV, Boada J, et al. Cell stress induces TDP-43 pathological changes associated with ERK1/2 dysfunction: implications in ALS. Acta Neuropathol. 2011; 122(3):259-70.

[15] Braun RJ, Sommer C, Carmona-Gutierrez D, Khoury CM, Ring J, Büttner S, et al. Neurotoxic 43-kDa TAR DNA-binding protein (TDP-43) triggers mitochondrion-dependent programmed cell death in yeast. J Biol Chem. 2011; 286(22):19958-72.

[16] Duan W, Li X, Shi J, Guo Y, Li Z, Li C. Mutant TAR DNAbinding protein-43 induces oxidative injury in motor neuron-like cell. Neuroscience. 2010; 169(4):1621-9.

[17] Schlesinger N. Dietary factors and hyperuricaemia. Curr Pharm Des 2005; 1:4133-4138.

[18] Garrel DR, Verdy M, PetitClerc C, Martin C, Brule D, Hamet P. Mild- and soy-protein ingestion: acute effect on serum uric acid concentration. Am J Clin Nutr 1991; $53: 665$.

[19] Hodgson EK, Fridovich I. Interaction of bovine erythrocyte superoxide dismutase with hydrogen peroxide. Chemiluminescence and peroxidation. Biochemistry 1975; 14(24):5299-5303.

[20] Choi HK, Liu S, Curhan G. Intake of purine-rich foods, protein, and dairy products and relationship to serum levels of uric acid: the third national health and nutrition examination survey. Arthrit is Rheum 2005; 52:283-9. 